\title{
Sunitinib as Neoadjuvant Chemotherapy in the Management of Metastatic Renal Cell Carcinoma Mimicking a Glomus Vagale Tumor in the Head and Neck: A Case Report and Review of Literature
}

\author{
Serkan Erkan ${ }^{1}$ Jessica Somner ${ }^{2}$ Gunesh P. Rajan ${ }^{1,3}$ \\ ${ }^{1}$ Department of Otolaryngology, Head and Neck Surgery, Fiona \\ Stanley Hospital, Murdoch, Western Australia, Australia \\ 2 Department of Otolaryngology, Head and Neck Surgery, University of \\ Western Australia, Nedlands, Western Australia, Australia \\ ${ }^{3}$ Department of Otolaryngology, Head and Neck Surgery, School of \\ Surgery, University of Western Australia, Murdoch, Western \\ Australia, Australia \\ J Neurol Surg Rep 2016;77:e77-e82. \\ Address for correspondence Gunesh P. Rajan, MD, DM, FMH, FRACS, \\ Department of Otolaryngology, Head and Neck Surgery, Fiona Stanley \\ Hospital, Murdoch, WA, 6150, Australia \\ (e-mail: Gunesh.Rajan@health.wa.gov.au).
}

\begin{abstract}
Keywords

- skull base metastasis

- renal cell carcinoma

- sunitinib

- cranial nerve palsy

- glomus vagale tumor

Background Metastatic renal cell carcinoma (RCC) of the head and neck with intracranial extension is rare and may pose difficulties to the diagnosis and management.

Method We describe a unique case of a 76-year-old man with a metastatic RCC to the neck and lateral skull base with intracranial extension presenting with Collet-Sicard syndrome 8 years after initial diagnosis. The radiologic features were consistent with the diagnosis of a glomus vagale tumor on the basis of clinical and radiologic features.

Results Despite radiotherapy, the intracranial extension progressed in size, resulting in early hydrocephalus. Sunitinib, a novel tyrosine kinase inhibitor, was instituted to treat the glomus vagale tumor with a marked reduction in tumor volume and resolution of the early hydrocephalus. The surgical resection of the tumor with its intracranial extension was achieved without additional postoperative morbidity. The histopathologic diagnosis surprisingly demonstrated metastatic RCC.

Conclusion We present a case of metastatic RCC to the head and neck region mimicking a glomus vagale tumor and describe the first use of sunitinib as a neoadjuvant chemotherapy to achieve a single-stage radical en bloc resection of the tumor mass.
\end{abstract}

\section{Introduction}

Renal cell carcinoma (RCC) is a highly malignant cancer with an unpredictable behavior of metastatic spread to various sites. ${ }^{1,2}$ About $15 \%$ of the RCC metastasis is located in the head and neck with thyroid gland and the sinonasal region being the most commonly affected sites. ${ }^{3-6}$ Metastatic tumors involving the skull base commonly originate from primary malignancies of the lung, breast, and prostate. ${ }^{1,7}$ An RCC metastasis extending into the skull base and posterior cranial fossa via hypoglossal foreman has not been reported so far, and skull base involvement is very rare with only two reported cases of glomus tumor mimic in the literature. ${ }^{8,9}$

The clinical symptoms of a lateral skull base involvement may present as paresis of cranial nerves IX, X, and XI known as jugular foramen syndrome. Additional involvement of the received

August 8, 2015

accepted after revision

January 3, 2016
DOI http://dx.doi.org/

10.1055/s-0036-1579629. ISSN 2193-6358. (c) 2016 Georg Thieme Verlag KG
Stuttgart · New York

License terms

(c) $(1) \$$ 
hypoglossal nerve is known as Collet-Sicard syndrome, which was the initial presentation of our case. As RCC has a tendency for early metastasis, these syndromes can be the first presentation preceding the symptoms of primary RCC. ${ }^{4,5,8,9}$ Conversely, the metastasis to the head and neck may develop long after the treatment of primary disease. ${ }^{8}$

We present a unique case of a 76-year-old man with an RCC metastasis to the upper neck extending into the skull base and posterior cranial fossa via jugular foreman. The lesion was initially diagnosed as a glomus vagale tumor due to the clinical symptoms and the typical radiological features. As the lesion was not deemed resectable due to the associated comorbidity, a trial with sunitinib was instituted with marked reduction in the size and extent of the various components of the tumor. As a result, the residual tumor mass was amenable to surgical resection.

This unique case is the first report describing the potential use of sunitinib as neoadjuvant chemotherapy to reduce the size and the extent of metastatic RCC ( $\mathrm{MRCC}$ ) disease to the head and neck in order to facilitate the total oncologic resection of such tumors without additional functional morbidity.

\section{Case Report}

A 76-year-old man presented to the ENT (ear-nose-throat) clinic at a tertiary center with a 6-month history of progressive dysphagia, dysphonia, and choking in December 2005. The examination revealed a right-sided atrophy of the tongue, paresis of the soft palate, and weakness of the right sternocleidomastoid and trapezius muscles. Laryngoscopic assessment demonstrated a fixed left vocal cord. The lower four cranial nerve dysfunction was typical of Collet-Sicard syndrome. He was an ex-smoker and had a history of right RCC treated overseas with a radical nephrectomy in 2000. At the same institution overseas he underwent a left-sided vocal cord medialization for a vocal cord paralysis in 2002 .

Magnetic resonance imaging (MRI) demonstrated a $50-\times 31-\times 24-\mathrm{mm}$ avidly enhancing mass with a "salt and pepper" appearance in the right carotid space extending from just below the jugular foramen to the level of carotid bifurcation (-Fig. 1). Considering the long-standing history of symptoms and the typical radiological features, the neuroradiologists were confident with the diagnosis of a right vagal paraganglioma as the lesion did not demonstrate the features of an mRCC. The multidisciplinary team (MDT) concluded that the lesion was very likely responsible for the vocal cord lesion detected and treated with a thyroplasty in 2002 and that its relatively benign, noninvasive behavior from 2002 to 2005 was not compatible with an mRCC. As a result, no biopsy was taken as per our paraganglioma workup protocol. Because of his worsening symptoms and his comorbidities, he underwent external beam radiotherapy with a total of $45 \mathrm{~Gy}$ in 25 fractions in May 2006, leading to a substantial clinical improvement of his swallow and speech, so that he was able to commence a full diet. Six months later, a follow-up MRI surprisingly revealed a progression of the lesion with involvement of the skull base and extension of the tumor through the hypoglossal foramen into the posterior cranial fossa measuring $10 \times 10 \times 11 \mathrm{~mm}$ (-Fig. 2). The intracranial component was treated with stereotactic radiotherapy in early 2007 with slight reduction in tumor size. Unfortunately, the symptoms recurred with multiple episodes of aspiration. Subsequent imaging in early 2009 revealed a significant growth of the intracranial component $(26 \times 25 \times 44 \mathrm{~mm})$ with compression of the brainstem and cerebellar hemisphere with vasogenic edema and early hydrocephalus (-Fig. 3). The tumor was deemed unresectable at the head and neck MDT meeting due to the risk of the significant peri- and postoperative mortality and morbidity. The medical oncologists suggested a trial of sunitinib on the basis of the reported use of sunitinib for the treatment of intra-abdominal paraganglioma in the medical literature. ${ }^{10-13}$ After IRB approval, the medical oncologists administered the sunitinib with a dosing regimen of $50 \mathrm{mg} / \mathrm{d}$ for 7 months. The dose was then reduced to $37.5 \mathrm{mg} / \mathrm{d}$ for 2 months due to side effects of hand-foot syndrome and nausea. After the first cycle of sunitinib, a follow-up MRI revealed a marked reduction in the extra- and intracranial components of the tumor ( - Fig. 4). A follow-up MRI 8 weeks post-sunitinib therapy, however, demonstrated an increase in tumor size with revascularization (-Fig. 5). Given the
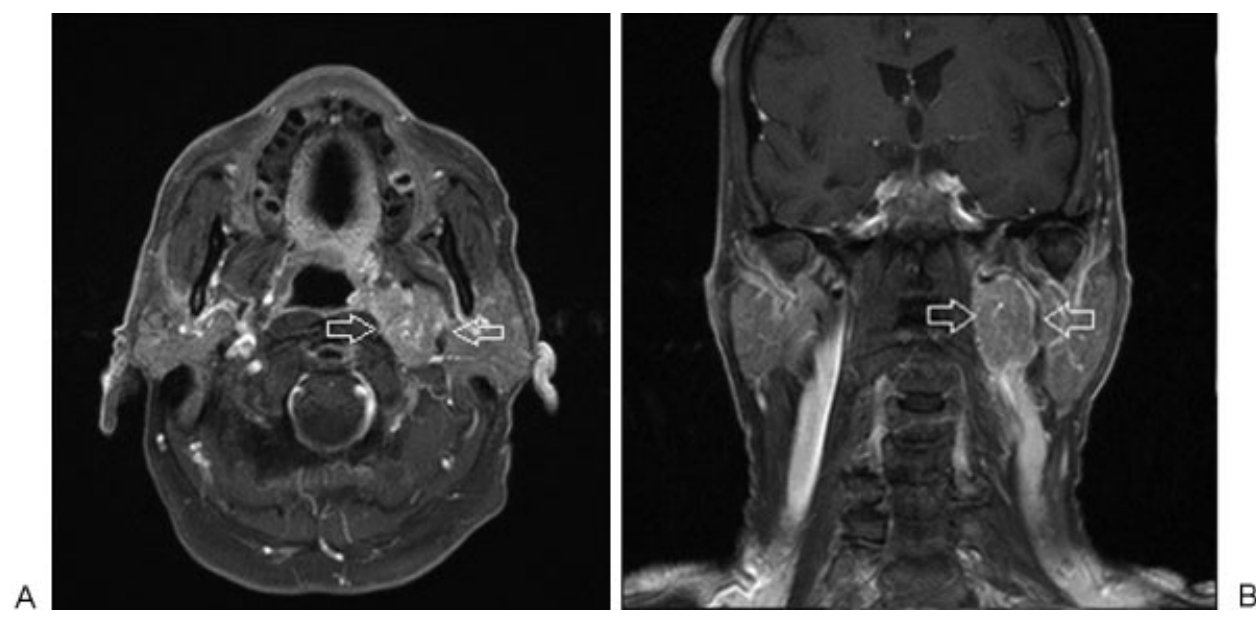

Fig. 1 T1-weighted MRI in December 2005 demonstrates a left carotid sheath soft tissue mass (A and B) with no definite intracranial extension. 

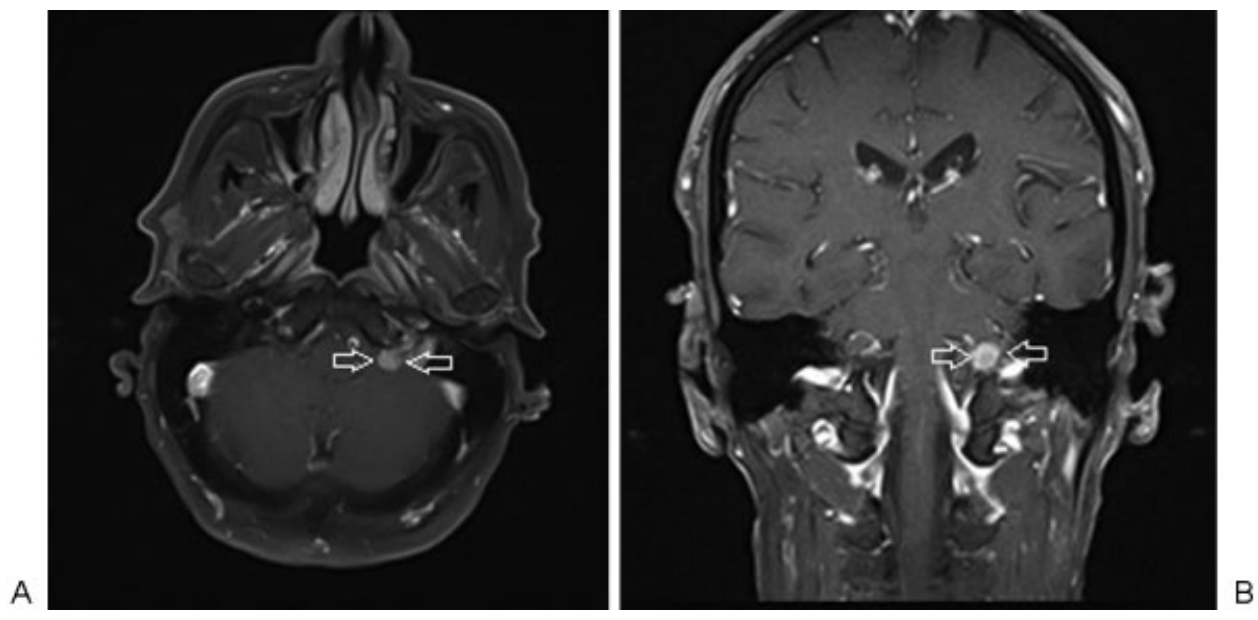

Fig. 2 T1-weighted MRI November 2006 demonstrating an enhancing posterior left cerebellopontine angle mass (A and B), the focus of an intracranial extension measuring $10 \times 10 \times 11 \mathrm{~mm}$.
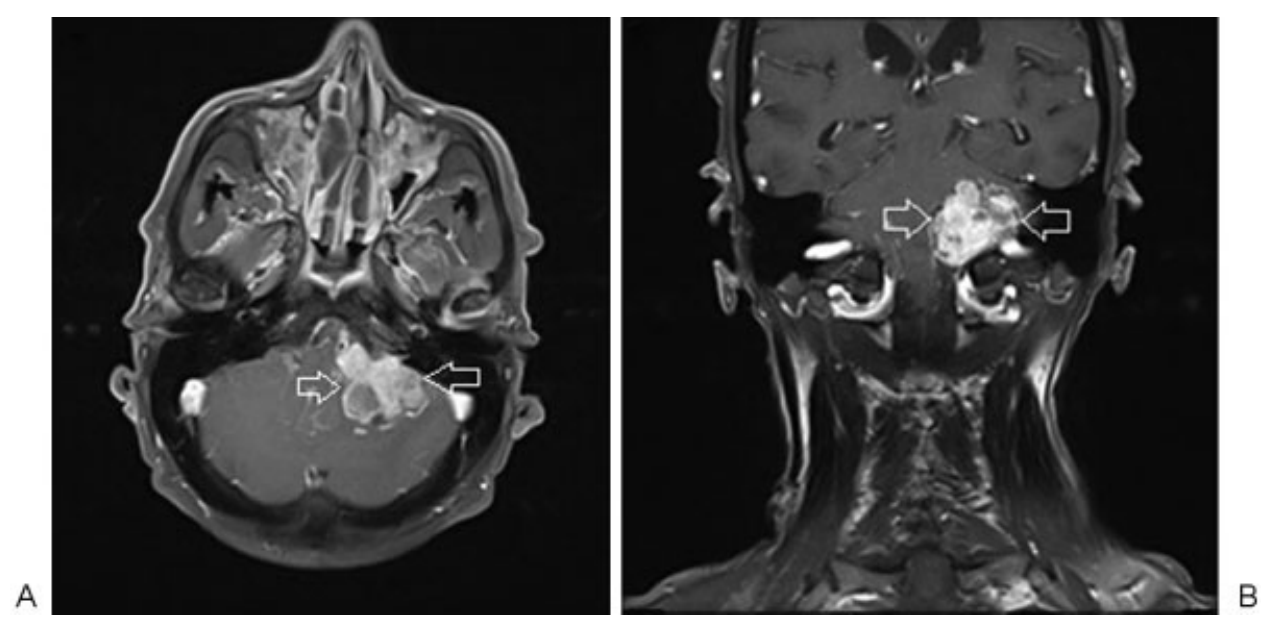

Fig. 3 T1-weighted MRI from September 2009 demonstrating further increase in the intracranial component now measuring $26 \times 25 \times 44$ mm (A and B), with early hydrocephalus.
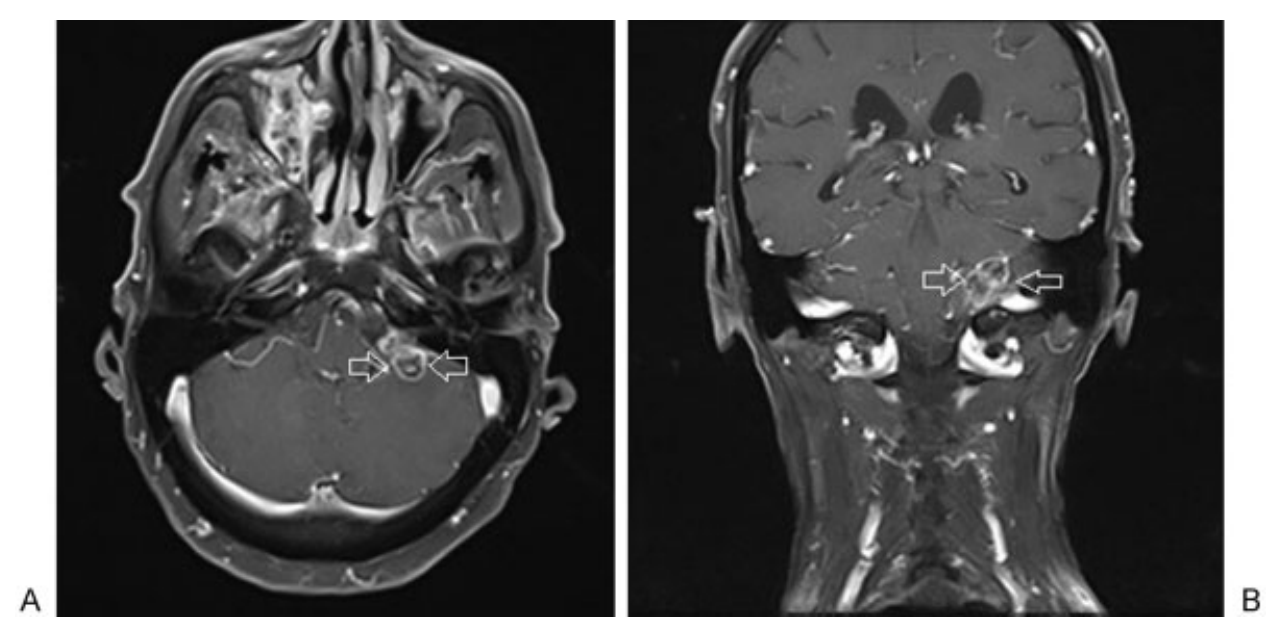

Fig. 4 T1-weighted MRI from March 2010, 6 months after commencement of sunitinib therapy. The left posterior fossa CPA tumor (A and B) now measures $19 \times 19 \times 26 \mathrm{~mm}$. 

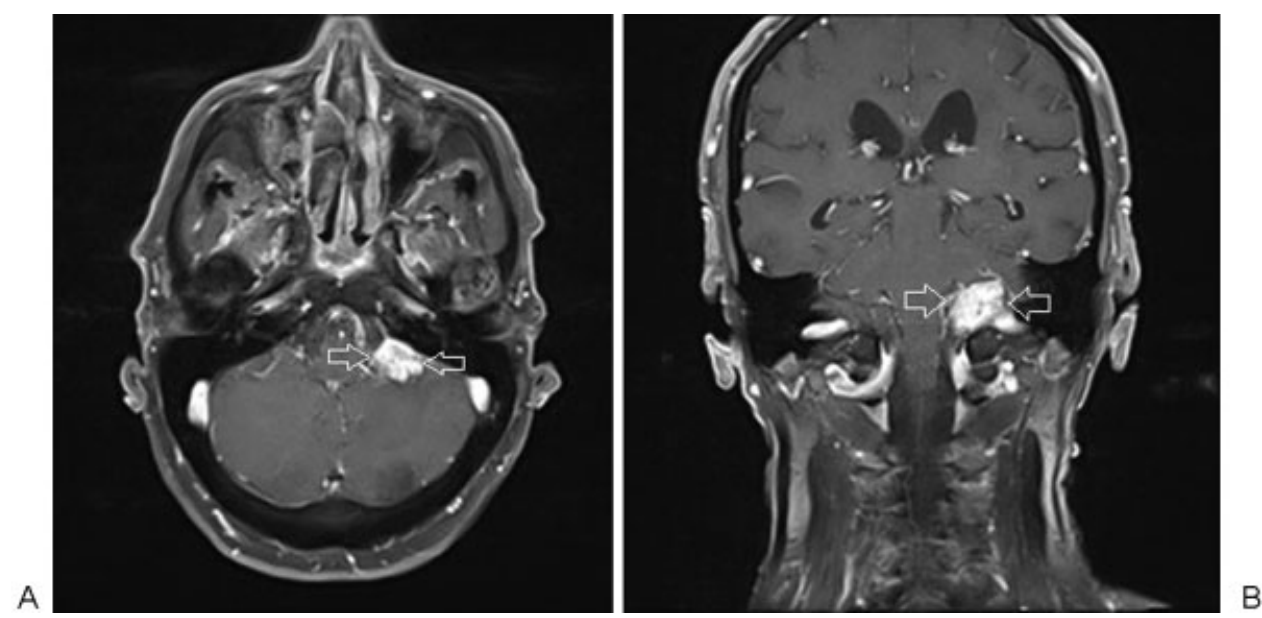

Fig. 5 T1-weighted MRI from August 2010, 8 weeks post sunitinib cessation showing interval progression of disease with increase in size and revascularization (A and $B)$.

resolution of brainstem and cerebellar compression postchemotherapy and imminent likelihood of recurrence of intracranial complications from the enlarging tumor volume, the head and neck MDT recommended surgical resection.

An en bloc resection of the tumor with its intracranial extension was performed using a combined transcervical, transsigmoidal, transjugular and type B infratemporal fossa approach with an uneventful postoperative course. To the surprise of the MDT, the histopathology revealed clear cell morphology consistent with an mRCC. Nine months after the surgery, the follow-up MRI showed no residual or recurrent tumor (-Fig. 6). However, the patient passed away 11 months later due to the complications of aspiration pneumonia.

\section{Discussion}

RCC constitutes only $3 \%$ of all adult malignancies and $90 \%$ of the renal malignancies, with clear cell morphology being the commonest subtype. ${ }^{1,4,5}$ It often occurs in the sixth decade of life with men having higher risk of developing RCC than women. ${ }^{14,15}$ The vast majority of the cases are shown to be sporadic while $5 \%$ of RCC are inherited. ${ }^{16,17}$ RCC has the one of the most unpredictable patterns of metastatic spread among all malignancies. ${ }^{1}$ The most common sites of metastasis are the lungs followed by the bone, liver, and subcutaneous tissue. $^{9,18,19}$ In approximately $30 \%$ of the cases, clinical presentation is secondary to the metastatic tumor rather than the primary malignancy. Metastasis to the head and neck region may present with a slowly enlarging neck mass, anosmia, epistaxis, or with cranial nerve paresis before the diagnosis of RCC is established. ${ }^{4,8,9}$

Symptoms of metastatic tumors to the skull base depend on the location, and as such, five distinct clinical presentations are identified. These are known as orbital, parasellar, middle fossa, jugular foramen, and occipital condyle syndromes. ${ }^{20}$

Jugular foreman syndrome is defined as the paresis of the cranial nerves IX, X, and XI, and is caused by the tumor invading through jugular foreman resulting in nerve compression. ${ }^{21}$ As in our case, involvement of lower four cranial nerves is known as Collet-Sicard syndrome. ${ }^{20}$ Its clinical symptoms include ipsilateral atrophy of the tongue, paresis of the soft palate, the absence of gag reflex, vocal cord
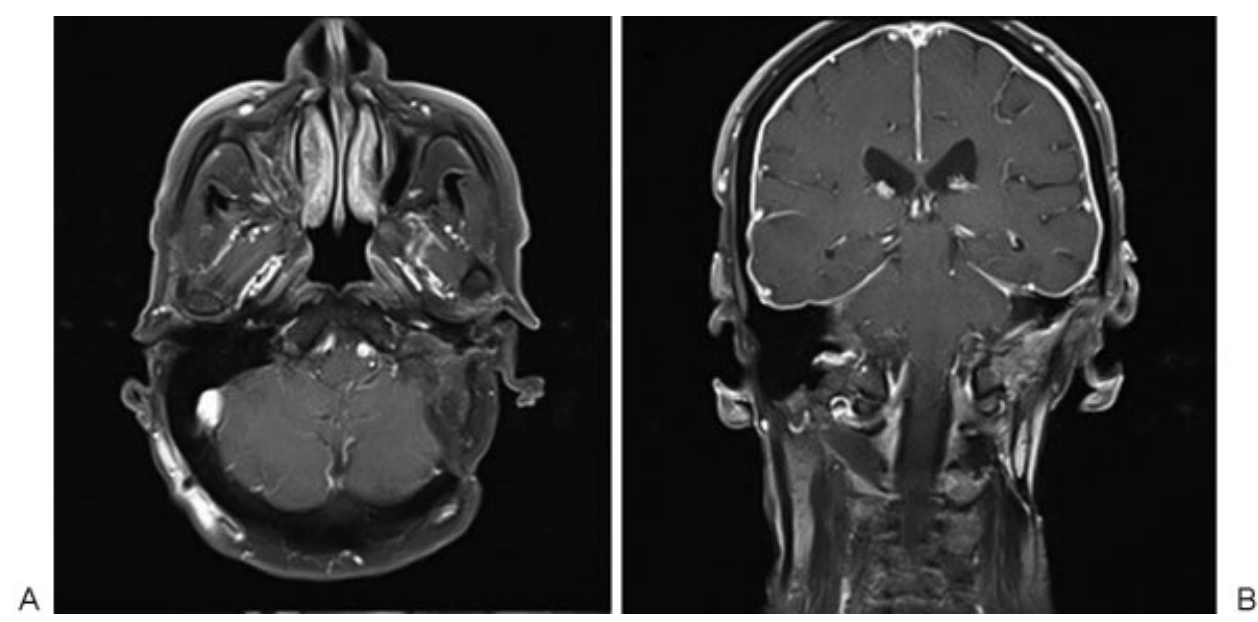

Fig. 6 MRI 9 months postoperative with no residual/recurrent skull base or posterior fossa tumor (A and B). 
palsy, ipsilateral weakness of the sternocleidomastoid, and trapezius muscles as well as contralateral deviation of the uvula. $^{20}$

The most common types of primary lateral skull base tumors include glomus tumors, schwannomas, and meningioma. ${ }^{22}$ Metastatic involvement of this region is very rare. In this case, the clinical history and the radiologic evidence of the avidly enhancing mass in the carotid space with anterior displacement of the carotid arteries and the typical "salt and pepper" appearance were both suggestive of a glomus vagale tumor with progression into the skull base and the posterior cranial fossa. Paraganglioma are usually not biopsied at our institution, especially if the neuroradiologists are confident with the diagnosis. Additionally the long-standing history was thought not to be compatible with the natural course of an mRCC. In the reported two cases of mRCC to the skull base mimicking a glomus tumor, palliative radiotherapy was administered as the patients had significant metastatic disease elsewhere within a short period of time. ${ }^{4-6,8,9}$ This case impressively demonstrates the reported "mimicry" and resemblance of RCC metastasis with glomus tumors of the head and neck. Hence, now we have learned that a high level of clinical suspicion for metastatic tumor to the head and neck is crucial in patients with a previous history of RCC.

The management of mRCC commonly involves combination of surgical resection, radiotherapy, chemotherapy with an angiogenesis inhibitor or a combination thereof. ${ }^{23}$ Metastatic tumors of the head and neck are often associated with functional morbidity and adverse functional outcomes. Radical oncologic resection is usually not possible as significant postoperative morbidity is often unavoidable due to the close proximity of the vital neurovascular structures in this complex location. In our case, sunitinib was administered by our medical oncologists to treat the suspected paraganglioma in analogy to the established treatment of abdominal paraganglioma with sunitinib. Because of the favorable response of the lesion to the sunitinib, a surgical removal was implemented, using a combined transsigmoidal, transjugular type B infratemporal fossa approach. This provided adequate exposure and direct access to the skull base and posterior fossa with excellent control of the jugular foramen and the carotid space allowing en bloc resection, and minimized the risk of postoperative neurovascular morbidity. ${ }^{24}$

There have been numerous studies regarding the use of sunitinib in mRCC but no reports specifically for the head and neck region with skull base or intracranial extension. This case illustrates that sunitinib can be used as neoadjuvant treatment to facilitate radical en bloc resection of the mRCC of the head and neck while preserving function and reducing surgical morbidity. The promising role of sunitinib in the management of head and neck RCC metastases merits further investigation. The role of sunitinib in the management of head and neck paraganglioma is yet to be determined.

\section{Conclusion}

Metastatic RCC to the head and neck region with extension into the skull base is very rare. Frequently, it is impossible to differentiate between an mRCC lesion and a skull base glomus tumor both clinically and radiologically. As such, tissue diagnosis is warranted for definite diagnosis. The unpredictable pattern of spread and clinical presentation of mRCC can escape initial diagnosis and present therapeutic challenges for clinicians. As a first, sunitinib was used as neoadjuvant chemotherapy to enable surgical resection of a head and neck RCC metastasis with extensive involvement of the skull base and posterior cranial fossa. The neoadjuvant potential and the local control provided by sunitinib open up new possibilities in the management of RCC metastasis to the head and neck with involvement of the skull base.

Financial Disclosure

None.

Conflict of Interest

None.

Note

Below manuscript has not been previously published in whole or in part or submitted elsewhere for review.

\section{References}

1 Ogunyemi O, Rojas A, Hematpour K, Rogers D, Head C, Bennett C. Metastasis of genitourinary tumors to the head and neck region. Eur Arch Otorhinolaryngol 2010;267(2):273-279

2 Wahner-Roedler DL, Sebo TJ. Renal cell carcinoma: diagnosis based on metastatic manifestations. Mayo Clin Proc 1997;72(10): 935-941

3 Miyamoto R, Helmus C. Hypernephroma metastatic to the head and neck. Laryngoscope 1973;83(6):898-905

4 Gottlieb MD, Roland JT Jr. Paradoxical spread of renal cell carcinoma to the head and neck. Laryngoscope 1998;108(9): 1301-1305

5 Pritchyk KM, Schiff BA, Newkirk KA, Krowiak E, Deeb ZE. Metastatic renal cell carcinoma to the head and neck. Laryngoscope 2002;112(9):1598-1602

6 Langille G, Taylor SM, Bullock MJ. Metastatic renal cell carcinoma to the head and neck: summary of 21 cases. J Otolaryngol Head Neck Surg 2008;37(4):515-521

7 Morvan JB, Veyrières JB, Mimouni O, Cathelinaud O, Allali L, Verdalle P. Clear-cell renal carcinoma metastasis to the base of the tongue and sphenoid sinus: two very rare atypical ENT locations. Eur Ann Otorhinolaryngol Head Neck Dis 2011; 128(2):91-94

8 Boileau MA, Grotta JC, Borit A, et al. Metastatic renal cell carcinoma simulating glomus jugulare tumor. J Surg Oncol 1987;35(3): 201-203

9 De Vos C, Gerard JM, Decat M, Gersdorff M. Metastatic renal cell carcinoma to the temporal bone: case report. B-ENT 2005;1(1): 43-46

10 Prochilo T, Savelli G, Bertocchi P, et al. Targeting VEGF-VEGFR pathway by sunitinib in peripheral primitive neuroectodermal tumor, paraganglioma and epithelioid hemangioendothelioma: three case reports. Case Rep Oncol 2013;6(1):90-97

11 Bourcier ME, Vinik AI. Sunitinib for the treatment of metastatic paraganglioma and vasoactive intestinal polypeptide-producing tumor (VIPoma). Pancreas 2013;42(2):348-352 
12 Joshua AM, Ezzat S, Asa SL, et al. Rationale and evidence for sunitinib in the treatment of malignant paraganglioma/pheochromocytoma. J Clin Endocrinol Metab 2009;94(1):5-9

13 Hahn NM, Reckova M, Cheng L, Baldridge LA, Cummings OW, Sweeney CJ. Patient with malignant paraganglioma responding to the multikinase inhibitor sunitinib malate. J Clin Oncol 2009; 27(3):460-463

14 Chow WH, Dong LM, Devesa SS. Epidemiology and risk factors for kidney cancer. Nat Rev Urol 2010;7(5):245-257

15 Ridge CA, Pua BB, Madoff DC. Epidemiology and staging of renal cell carcinoma. Semin Intervent Radiol 2014;31(1):3-8

16 Vanharanta S, Buchta M, McWhinney SR, et al. Early-onset renal cell carcinoma as a novel extraparaganglial component of SDHBassociated heritable paraganglioma. Am J Hum Genet 2004;74(1): 153-159

17 Williamson SR, Eble JN, Amin MB, et al. Succinate dehydrogenasedeficient renal cell carcinoma: detailed characterization of 11 tumors defining a unique subtype of renal cell carcinoma. Mod Pathol 2015;28(1):80-94
18 Koutnouyan HA, Rumore GJ, Kahn JM. Skull metastasis from renal cell carcinoma. Case report and literature review. Ann Otol Rhinol Laryngol 1998;107(7):598-602

19 Yeh HC, Yang SF, Ke HL, Lee KS, Huang CH, Wu WJ. Renal cell carcinoma presenting with skull metastasis: a case report and literature review. Kaohsiung J Med Sci 2007;23(9):475-479

20 Bone I, Hadley DM. Syndromes of the orbital fissure, cavernous sinus, cerebello-pontine angle, and skull base. J Neurol Neurosurg Psychiatry 2005;76(Suppl 3):iii29-iii38

21 Robbins KT, Fenton RS. Jugular foramen syndrome. J Otolaryngol 1980;9(6):505-516

22 Löwenheim H, Koerbel A, Ebner FH, Kumagami H, Ernemann U, Tatagiba M. Differentiating imaging findings in primary and secondary tumors of the jugular foramen. Neurosurg Rev 2006; 29(1):1-11, discussion 12-13

23 Motzer RJ, Rini BI, Bukowski RM, et al. Sunitinib in patients with metastatic renal cell carcinoma. JAMA 2006;295(21):2516-2524

24 Browne JD, Fisch U, Valavanis A. Surgical therapy of glomus vagale tumors. Skull Base Surg 1993;3(4):182-192 